Military Technical College

Kobry Elkobbah,

Cairo, Egypt

May $29-31,2012$ $6^{\text {th }}$ International Conference

on Mathematics and

Engineering Physics

(ICMEP-6)

\title{
EFFECT OF POROSITY ON THE FLOW OF A VISCOELASTIC FLUID OVER A STRETCHING SHEAT
}

\author{
Hazem Ali Attia ${ }^{1} \quad$ Karem Mahmoud Ewis Ibrahim Hamdy Abd Elmaksoud \\ Department of Engineering Mathematics and Physics, \\ Faculty of Engineering, Fayoum University, \\ El-Fayoum-63415, Egypt
}

\begin{abstract}
The flow through a porous medium of a non-Newtonian viscoelastic fluid past a stretching sheet is examined where the porosity of the medium is assumed to obey Darcy's model. An analytical solution of the governing non-linear boundary layer equation is obtained. The effect of the porosity of the medium and the characteristics of the non-Newtonian fluid on the flow is investigated.
\end{abstract}

\section{Introduction}

The flow of a viscous incompressible fluid over a moving surface otherwise at rest has several engineering applications in polymer processing. Crane [1] studied the steady two-dimensional boundary layer flow caused by a stretching sheet whose velocity varies linearly with the distance from a fixed point on the sheet. The extension of the problem to the case of non-Newtonian fluids has been given by [2-10]. Chiam [2] and Anderson and Dandapat [3] have considered the motion of micropolar and power-law fluids, respectively whereas the analysis of viscoelastic fluids was given by others [4-10]. Unlike the inelastic power-law model, the Rivlin-Ericksen fluid studied by Siddappa and Khapate [4] and Walters' liquid B' considered by Siddappa and Abel [6] both exhibit normal-stress differences in simple shear flows [10]. Rajagopal, Na and Gupta [5] studied the effects of viscoelasticity on the flow of a second-order fluid and obtained the same governing boundary layer equation as that in [6], [7] and [10]. The flow of electrically conducting viscoelastic fluid past a stretching sheet in the presence of an external uniform magnetic field was studied in [11].

In the present paper, the effect of the porosity of the medium on the flow of a viscoelastic fluid past a stretching sheet is analyzed. The flow through a porous medium deals with the analysis in which the differential equation governing the fluid motion is based on the Darcy's law which accounts for the drag exerted by the porous medium [1214]. An analytical solution of the governing non-linear boundary layer equation is obtained, showing that the porous medium has the same effect on the flow as the characteristics of the non-Newtonian viscoelastic fluid.

\footnotetext{
19ah1113@yahoo.com
} 
Military Technical College

Kobry Elkobbah,

Cairo, Egypt

May $29-31,2012$ $6^{\text {th }}$ International Conference

on Mathematics and

Engineering Physics

(ICMEP-6)

\section{Formulation of the Problem}

The flow through a porous medium of an incompressible viscoelastic fluid over a flat and impermeable elastic sheet is studied. The fluid (Walters'liquid B'), which is confined to the half space $y>0$ above the sheet, obeys the rheological equations of state due to Walters [15-16]. The sheet is stretched with a velocity which varies linearly with the distance from the fixed origin $x=0$ by applying two equal and opposite forces along the $x$-axis. The resulting motion of the otherwise fluid at rest is thus caused solely by the moving sheet. The steady two-dimensional boundary layer equations for Walters'liquid B' were derived by Beard and Walters [17] to first order in elasticity which means for short memory fluids with short relaxation times. Following [16] the equations governing the viscoelastic boundary layer flow along a stretching sheet take the form

$\frac{\partial u}{\partial x}+\frac{\partial v}{\partial y}=0$

$\rho\left(u \frac{\partial u}{\partial x}+v \frac{\partial u}{\partial y}\right)=-\frac{\mu}{K} u+\mu \frac{\partial^{2} u}{\partial y^{2}}-\alpha\left(u \frac{\partial^{3} u}{\partial x \partial y^{2}}+v \frac{\partial^{3} u}{\partial y^{3}}+\frac{\partial u}{\partial x} \frac{\partial^{2} u}{\partial y^{2}}-\frac{\partial^{2} u}{\partial x \partial y} \frac{\partial u}{\partial y}\right)$

with the boundary conditions

$y=0: u=c x, v=0, x \geq 0$,

$y \rightarrow \infty: u \rightarrow 0, x \geq 0$,

where $u$ and $v$ are the velocity components in the $x$ and $y$ directions, respectively, $\rho$ is the density, $\mu$ is the limiting viscosity at small rates of shear, $\alpha$ is the first moment of the distribution function of relaxation times, and $K$ is the Darcy permeability [12-14].

\section{Analytical Solution}

Following the procedure discussed in [11], we introduce the new dimensionless variables $f$ and $\eta$ such that

$$
\begin{aligned}
& \psi=(c \mu / \rho)^{1 / 2} x f(\eta) \\
& \eta=(c \rho / \mu)^{1 / 2} y
\end{aligned}
$$

The velocity components $u$ and $v$ are then related to the physical stream function $\psi$ by

$$
\begin{aligned}
& u=\partial \psi / \partial y=c x f^{\prime}(\eta) \\
& v=-\partial \psi / \partial x=-(c \mu / \rho)^{1 / 2} f(\eta)
\end{aligned}
$$

Substituting Eq. (5) in Eqs. (1)-(3) we get the fourth-order ordinary differential equation 
Military Technical College

Kobry Elkobbah, Cairo, Egypt

May $29-31,2012$ $6^{\text {th }}$ International Conference

on Mathematics and

Engineering Physics

(ICMEP-6)

$$
f^{\prime \prime \prime}-f^{\prime 2}+f f^{\prime \prime}=N f^{\prime}+k\left(2 f f^{\prime \prime \prime}-f^{\prime \prime 2}-f f^{\prime \prime \prime \prime}\right),
$$

with the transformed boundary conditions

$\eta=0: f^{\prime}=1, f=0$

$\eta \rightarrow \infty: f^{\prime} \rightarrow 0$

The terms on the right side of Eq. (6) represent the porosity and viscoelastic forces where $N=v /(c K)$ and $k=c \alpha / \mu$ are the porosity and elastic parameters, respectively. The latter represents a measure of the relative importance of elastic and viscous effects and can thus be identified with the Weissenberg number [10]. Recently in a similar formulation but in the non-porous case, Dandapat and Gupta [9] found that a function of the form

$f(\eta)=\frac{1}{\beta}\left(1-e^{-\beta \eta}\right), \beta>0$

satisfies the complete set of boundary conditions in Eq. (7). It can shown checked that Eq. (8) is a solution of the highly non-linear differential equation (6) if

$\beta=\left(\frac{1+N}{1-k}\right)^{1 / 2}$.

Therefore, the solutions for the velocity components become

$u=c x e^{-\beta \eta}, \quad v=-(c \mu / \rho)^{1 / 2}\left(1-e^{-\beta \eta}\right) / \beta$

This analytical solution includes the combined effects of viscoelastic and porosity forces. The analytical solution derived independently in [6] and [9] is obtained for $N=0$.

\section{Discussion}

Following the procedure discussed in [11], far away from the stretching sheet the velocity field in Eq. (10) acts as

As $\eta \rightarrow \infty: u \rightarrow 0, v \rightarrow-(c \mu / \rho)^{1 / 2} / \beta$

Therefore, the flow outside the boundary layer becomes uniform and is directed perpendicularly to the sheet. The boundary layer thickness $\delta$ defined as the distance from the sheet at which the velocity component $u$ has been reduced to one percent of the velocity $c x$ of the stretching surface, and is expressed as [11]

$\delta=\ln 100(\mu / \rho c)^{1 / 2} / \beta$. 
Military Technical College

Kobry Elkobbah,

Cairo, Egypt

May $29-31,2012$ $6^{\text {th }}$ International Conference

on Mathematics and

Engineering Physics

(ICMEP-6)

Thus, $\delta$ is constant and independent of $x$ for any combination of the parameters $N$ and $k$.

The dimensionless wall friction coefficient $c_{f}$ can be given as

$c_{f}=\frac{-\tau_{w} / \rho}{(c x)^{2}}=-f^{\prime \prime}(0) \operatorname{Re}_{x}^{-1 / 2}=\beta \operatorname{Re}_{x}^{-1 / 2}$

where $\operatorname{Re}_{x} \equiv \rho c x^{2} / \mu$ is a local Reynolds number based on the velocity $c x$ of the stretching sheet.

An essential feature of the above analytical results is that the viscoelastic and porosity effects are combined into the single parameter $\beta \geq 1$ defined in Eq. (9). Since $N \geq 0$ and $0 \leq k<<1$ it can be concluded that an increase of the porosity has the same influence on the flow field as increased viscoelasticity [11]. According to Eqs. (10)-(13) the main effects of elasticity and a porous medium are to reduce the velocity within the boundary layer and the external velocity normal to the sheet and also to reduce the boundary layer thickness while increasing the skin friction.

\section{References}

1. L.J. Crane, Flow past a stretching plate. ZAMP 21, 645-647 (1970).

2. T.C. Chiam, Micropolar fluid flow over a stretching sheet. ZAMM 62, 565-568 (1982).

3. H.I. Andersson and B.S. Dandapat, Flow of a power-law fluid over a stretching sheet. Stability. Appl. Anal. Continuous Media 1, 339-347 (1991).

4. B. Siddappa and B.S. Khapate, Rivlin-Ericksen fluid flow past a stretching plate. Rev. Roum. Sci. Techn.-Mec. Appl. 21, 497-505 (1976).

5. K.R. Rajagopal, T.Y. Na and A.S. Gupta, Flow of a viscoelastic fluid over a stretching sheet. Rheol. Acta 24, 213-215 (1984).

6. B. Siddappa and S. Abel, Non-Newtonian flow past a stretching plate. ZAMP 36, 890892 (1985).

7. B. Siddappa and S. Abel, Visco-elastic boundary layer flow past a stretching plate with suction and heat transfer. Rheol. Acta 25, 319-320 (1986).

8. N.M. Bujurke, S.N. Biradar and P.S. Hiremath, Second-order fluid flow past a stretching sheet with heat transfer. ZAMP 38, 653-657 (1987). 
Military Technical College

Kobry Elkobbah,

Cairo, Egypt

May $29-31,2012$

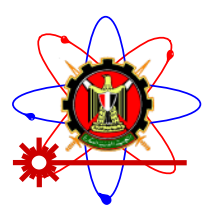

$6^{\text {th }}$ International Conference

on Mathematics and

Engineering Physics

(ICMEP-6)

9. B.S. Dandapat and A.S. Gupta, Flow and heat transfer in a viscoelastic fluid over a stretching sheet. Int. J. Non-Linear Mech. 24, 215-219 (1989).

10. N. Ahmed, G.S. Patel and B. Siddappa, Visco-elastic boundary layer flow past a stretching plate and heat transfer. ZAMP 41, 294-298 (1990).

11. H.I. Andersson, MHD flow of a viscoelastic fluid past a stretching surface. Acta Mech. 95, 227-230 (1992).

12. D.D. Joseph, D.A. Nield, G. Papanicolaou, "Nonlinear equation governing flow in a staturated porous media", Water Resources Research, 18(4), 1049-1052 (1982).

13. D.B. Ingham, and I. Pop, Transport phenomena in porous media. Pergamon, Oxford (2002).

14. A.R.A. Khaled and K. Vafai, "The role of porous media in modeling flow and heat transfer in biological tissues", Int. J. Heat Mass Transf., 46, 4989-5003 (2003).

15. K. Walters, The motion of an elastico-viscous liquid contained between coaxial cylinders (II). Q.J. Mech. Appl. Math. 13, 444-461 (1960).

16. K. Walters, Non-Newtonian effects in some elastico-viscous liquids whose behaviour at small rates of shear is characterized by a general linear equation of state. Q.J. Mech. Appl. Math. 15, 63-76 (1962).

17. D.W. Beard and K. Walters, Elastico-viscous boundary layer flows. I. Two dimensional flow near a stagnation point. Proc. Cambr. Phil. Soc. 60, 667-674 (1964). 